\title{
Uncertainty of Positioning and Displacement Measurements in Quantum and Thermal Regimes
}

\author{
Silvano Donati, Fellow, IEEE, Cheng-Yen Chen, and Chih-Chung Yang, Senior Member, IEEE
}

\begin{abstract}
We analyze the performance of position-sensing devices and of distance- or displacement-measuring instruments, and we find that the ultimate uncertainty at the quantum limit of detected signal is given by the same expression in all cases, namely, a characteristic length $L_{c}$ divided by $\sqrt{ } N_{\mathrm{ph}}$, the square root of the number of photons detected in the time interval of the measurement. We derive the expression of the length $L_{c}$ for well-known position-sensing devices (the quadrant photodiode and the position-sensing detector) and for several measuring instruments (pulsed and sine-wave-modulated rangefinders, triangulation telemeter, laser interferometer, and the optical rule). We also extend the analysis of the uncertainty results to the thermal regime case of detection, i.e., when the detector dark current and preamplifier noises are dominant with respect to quantum noise.
\end{abstract}

Index Terms-Instrumentation, interferometry, laser range finder, measurement, metrological instrumentation, metrology, optical instruments.

\section{INTRODUCTION}

A S IS well known, the uncertainty of position-sensing devices, as well as that of dimensional measuring instruments, is a crucial parameter in a number of applications ranging from industrial manufacturing and construction works to avionics and space [1]-[4].

The ultimate uncertainty at the quantum limit of signal detection has been analyzed by several authors [4]-[12], who also treated the case of squeezed states [7] and extended the results to the intervening atmospheric scintillation [8]. Thus, the quantum-limited dependence on $L_{c} / \sqrt{ } N_{\text {ph }}$ has already been reported in a few special cases of interest [4], [7]-[9].

In this paper, we present a comprehensive analysis of the uncertainty of both position-sensing and displacement-measuring instruments. We also extend the results to the more realistic case of a thermal noise and background or dark current noise added to the quantum noise of detected photons.

In addition, we consider in detail several practical cases of positioning or displacement measurements and derive for each of them the applicable value of the characteristic length.

Manuscript received December 8, 2005; revised January 4, 2007.

S. Donati was with the Graduate Institute of Electro-Optical Engineering, National Taiwan University, Taipei 106, Taiwan, R.O.C. He is now with the Department of Electronics, University of Pavia, 27100 Pavia, Italy (e-mail: silvano.donati@ieee.org).

C.-Y. Chen and C.-C. Yang are with the Graduate Institute of Electro-Optical Engineering, National Taiwan University, Taipei 106, Taiwan, R.O.C. (e-mail: d92941001@ntu.edu.tw; ccy@cc.ee.ntu.edu.tw).

Digital Object Identifier 10.1109/TIM.2007.895584
This paper is organized as follows. In Section II, we derive the uncertainty of position-sensing photodiodes, first at the quantum limit, and then, we extend the result to the more realistic case of intervening thermal noise. We bring the results into a unified expression of the type $L_{c} / \sqrt{ } N_{\text {ph }}$ by calculating in each case the appropriate characteristic length $L_{c}$. In Section III, we recall the results about the uncertainty of distancemeasuring instruments. Then, in Section IV, we draw conclusions.

\section{Position Sensing}

The quadrant photodiode (or Q-PD) has been the forerunner in position sensing [3], [4] along with rotating-reticle photodetectors [3] since the early times of the technique. It is still widely used [8]-[12] because it is fast, accurate, and straightforward to use, although its linearity is not so good as the one provided by the more modern position-sensing detectors (PSDs) [4].

In the Q-PD, we use the sum of signals coming from left- and right-side quadrants to generate the position-sensing deviations relative to the $X$ and $Y$ coordinates of spot center. As illustrated in Fig. 1, $L=S_{1}+S_{3}$ and $R=S_{2}+S_{4}$ are the $X$-coordinate signals. When $L$ is high, the spot is on the "left" (or $X<0$ ), and when $R$ is high, it is on the "right" (or $X>0$ ). Comparing $L$ and $R$, we can tailor an error signal suitable for the control loop action of the servo system committed to the alignment operation.

In particular, it is commonplace to subtract $L$ and $R$ to generate a signal $\xi=R-L$, which passes through zero at $X=0$, is about linear around $X \approx 0$ for a range on the order of the optical spot size (Fig. 1) and saturates to \pm 1 when $X$ is far away from zero [4].

Analogously, two signals $U=S_{1}+S_{2}$ and $D=S_{3}+S_{4}$ define the $Y$-coordinate, and their difference $\zeta=U-D$ is the signal for positioning along the $y$-axis.

Uncertainty of positioning is, of course, relative to both axes $X$ and $Y$. However, we may take advantage of the symmetry of the problem and restrict ourselves to consider the monodimensional case, the behavior along $X$, for example, avoiding unnecessary complications in deriving the results.

In the next section, we first analyze the uncertainty of positioning of the Q-PD at the quantum limit. Following that, we will extend the results to the PSD and to the case of electronic noise. 


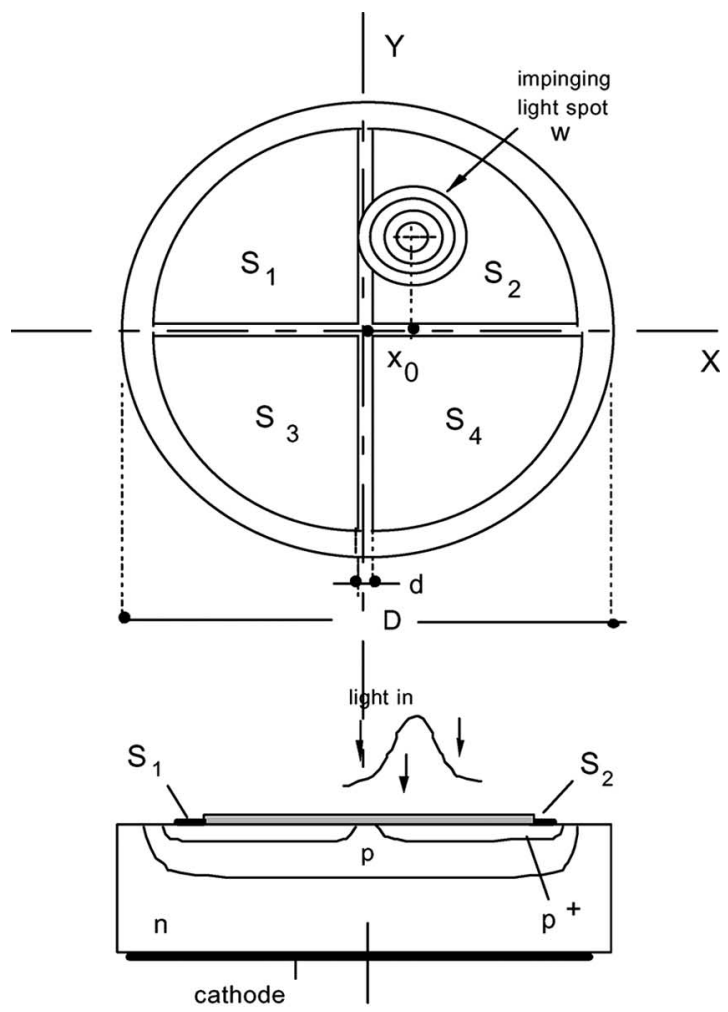

Now, in terms of the power density $p(x)$ falling on the quadrant active area, the total power $P$ being photo-detected is the integral of $p(x)$ over the collecting aperture of the quadrant.

Assuming the Gaussian as a reasonably general model for the power density distribution of the light spot and letting the light spot centered at coordinate $x_{0}$, we can write

$$
\begin{aligned}
p(x) & =P_{0} \operatorname{gauss}(x, w) \\
& =P_{0}(2 \pi)^{-1 / 2} w^{-1} \exp -\left[\left(x-x_{0}\right)^{2} / 2 w^{2}\right]
\end{aligned}
$$

where $w$ is the standard deviation (or rms value) of the Gaussian distribution, and $P_{0}$ is the total power carried by the spot. With this notation and with reference to Fig. 1 (top), we can write the photodiode signals as

$$
\begin{aligned}
& R=\int_{+d / 2}^{+\infty} p\left(x-x_{0}\right) d x=P_{0}\left[1-\operatorname{erf}\left(d / 2-x_{0}, w\right)\right] \\
& L=\int_{-\infty}^{-d / 2} p\left(x-x_{0}\right) d x=P_{0} \operatorname{erf}\left(-d / 2-x_{0}, w\right)
\end{aligned}
$$

where $d$ is the thickness of the dead band separating the quadrants (Fig. 1), and erf is the standard error function defined as

$$
\operatorname{erf}(x, w)=(2 \pi)^{-1 / 2} w^{-1} \int_{-\infty}^{x} \exp -\left(\kappa^{2} / 2 w^{2}\right) d \kappa .
$$

The coordinate signal then follows as $\xi=R-L$. From the above equations, we get for $\xi$ the following equation:

$$
\xi=P_{0}\left[1-\operatorname{erf}\left(d / 2-x_{0}, w\right)-\operatorname{erf}\left(-d / 2-x_{0}, w\right)\right]
$$

Fig. 1. Front view (top) and cross section (middle) of a Q-PD receiving a light spot for alignment; (bottom) graph of typical $X$-coordinate signals $R$ and $L$ (in the case of a small gap compared to spot size $d \ll w$ ).

\section{A. Analysis at the Quantum Noise Limit}

The positioning uncertainty of the sensor can be defined as the rms uncertainty of first kind [4], which is associated with the measurand (position), as the result of fluctuations affecting the measurement process.

The ultimate uncertainty left over when we are able to eliminate all other causes of fluctuations is that associated with the quantum nature (in the sense of first quantization) of the optical field, and we call the associated uncertainty as the quantumnoise limit.

The positioning uncertainty of the coordinate $X$ is defined as $\sigma_{x}=\left\langle(x-\langle x\rangle)^{2}\right\rangle^{1 / 2}$, where $\langle\ldots\rangle$ is the ensemble averaging operator.

The position signal supplied by the quadrant detector is $\xi=R-L$ (or $\zeta=U-D$ for the $y$-coordinate). On their turn, signals $R$ and $L$ are those supplied by the quadrant sections of the photodiode in the form of a photo-generated current $I_{\mathrm{ph}}=\varsigma P$ following the detection of optical power $P$ by the quadrant, $\varsigma=e \eta / h \nu$ being the spectral sensitivity [5].

and, for small $x_{0}$ and $d / 2(\ll w)$, developing (3) at the first order in $x_{0}$

$$
\begin{aligned}
\xi= & P_{0}(2 \pi)^{-1 / 2} w^{-1} \\
& \times\left\{\exp -\left[\left(-x_{0}+d / 2\right)^{2} / 2 w^{2}\right]\right\}\left(x_{0}-d / 2\right) \\
& -P_{0}(2 \pi)^{-1 / 2} w^{-1} \\
& \times\left\{\exp -\left[\left(-x_{0}-d / 2\right)^{2} / 2 w^{2}\right]\right\}\left(-x_{0}-d / 2\right) \\
\approx & P_{0}(2 \pi)^{-1 / 2} w^{-1} \\
& \times\left[\exp -x_{0}^{2} / 2 w^{2}\right] \exp -\left(d^{2} / 8 w^{2}\right) 2 x_{0} .
\end{aligned}
$$

Equation (4) tells us that for small $x_{0}$, signal $\xi$ is indeed proportional to the deviation $x_{0}$ of the light spot position from the zero of the Q-PD sensor coordinate $(x=0)$ and also shows the reduction factor due to the finite gap $d$.

We can now write the rms uncertainty in positioning, due to the fluctuations of the detected power, as

$$
\sigma_{x}^{2}=\sigma_{\xi}^{2} /\left|\partial \xi / \partial x_{0}\right|^{2}
$$

Here, we have used the principle of linear regression [13] by which, at the first order, the deviations $\Delta x_{0}$ and $\Delta \xi$ are 
connected by their partial derivative (the linear regression hypothesis is always satisfied if quantities are small). Assuming that we are operating in fair experimental conditions, that is with $x_{0}$ small and $d \ll w$, so that the exponential terms in (4) can be taken equal to 1 , the derivative is calculated as

$$
\partial \xi / \partial x_{0}=P_{0}(2 \pi)^{-1 / 2} w^{-1} 2 .
$$

About variance $\sigma_{\xi}^{2}$, we shall note that $\xi$ is the difference of signals $R$ and $L$, each of which is affected by a shot-noise fluctuation uncorrelated to that of the other term. Therefore, their individual variances shall be added:

$$
\sigma_{\xi}^{2}=\sigma_{L}^{2}+\sigma_{R}^{2} .
$$

Now, each term $\sigma_{L}^{2}, \sigma_{R}^{2}$ is written as the shot-noise variance associated with the power detected in the section $2 h \nu P_{L} B$ or $2 h \nu P_{R} B$, where $B$ is the bandwidth of observation (or of measurement) of the fluctuation. As it is well known, the shot-noise variance comes as a consequence of the Poisson distribution in the number of detected photons, which is a good approximation for most of the sources found in applications (see [4, Sec. 8.6]).

Considering that the sum of powers collected by quadrants gives just the total beam power $P_{L}+P_{R}=P_{0}$ and using this result in (4)-(6), we get

$$
\begin{aligned}
\sigma_{x}^{2} & =2 h \nu P_{0} B /\left[P_{0}^{2}(\pi / 2)^{-1} w^{-2}\right] \\
& =w^{2}(\pi / 2) 2 h \nu B / P_{0} \\
& =w^{2}(\pi / 2) /(\mathrm{S} / \mathrm{N})^{2} .
\end{aligned}
$$

The last expression, involving the signal-to-noise ratio associated with the measurement of a signal of amplitude $P_{0}$, i.e.,

$$
(\mathrm{S} / \mathrm{N})^{2}=P_{0} / 2 h \nu B
$$

has been already reported [[8]].

In (8), we may note that $P_{0} / h \nu$ is the rate at which photons are collected by the sensor. If we multiply this quantity by the observation time $T$, we get the number of photons $N_{\mathrm{ph}}=T P_{0} / h \nu$ utilized in the measurement. Time $T$ can be expressed in terms of bandwidth as $T=1 / 2 B$ in view of Nyquist theorem. Rearranging (8) accordingly, we get, for the rms uncertainty, the following equation:

$$
\sigma_{x}=(\pi / 2)^{1 / 2} w / \sqrt{ } N_{\mathrm{ph}}=m_{f} L_{c} / \sqrt{ } N_{\mathrm{ph}} .
$$

The result displays a dependence of length over square root of number of photons. In this case, the characteristic length $L_{c}$ is equal to the rms width of the Gaussian distribution, and the multiplication factor is $m_{f}=(\pi / 2)^{1 / 2}$. Of course, we may have got the multiplication factor equal to one, defining the Gaussian rms width $w(\pi / 2)^{1 / 2}$ instead of $w$. However, we prefer to avoid unnecessary burden in defining the characteristic length and retain the multiplication factor as it turns out from the calculation, which is a quantity never much different from unity.

Let us also consider another refinement about $\mathrm{S} / \mathrm{N}$ ratio and $N_{\mathrm{ph}}$ in the above expressions and in those to follow. Using an ideal photodetector with unity quantum efficiency $(\eta=1)$, these quantities are indifferently referred to collected photons or to detected photoelectrons, whereas in a real photodetector with $\eta<1$, we get a signal $I_{\mathrm{ph}}=\varsigma P_{0}[\varsigma=e \eta / h \nu$ being the spectral sensitivity], and a shot noise $\sigma_{I}^{2}=2 e I_{\mathrm{ph}} B$, where the signal-to-noise ratio in current $(\mathrm{S} / \mathrm{N})_{I}^{2}$ is found as

$$
\begin{aligned}
(\mathrm{S} / \mathrm{N})_{I}^{2} & =I_{\mathrm{ph}} / 2 e B \\
& =(e \eta / h \nu) P_{0} / 2 e B \\
& =\eta P_{0} / 2 h \nu B \\
& =\eta(\mathrm{S} / \mathrm{N})_{P}^{2}
\end{aligned}
$$

where definition (8a) has been used. Thus, the $\mathrm{S} / \mathrm{N}$ ratio for currents, which are the actual quantities available at the output of the photodetector, is $\sqrt{ } \eta$ times that for optical powers.

Accordingly, in all expressions of uncertainty, we shall understand a factor $\eta^{1 / 2}$ included in the multiplying factor $m_{f}$. For the same reason, $N_{\mathrm{ph}}$ is the number of detected photons (or photoelectrons) for $\eta=1$, whereas we shall take $\eta N_{\mathrm{ph}}$ as the number of photoelectrons available for the measurement if our detector has subunitary quantum efficiency.

Before proceeding, let us consider the uncertainty of PSD (or PSD photodiode, [4]). In this device, light collected at the point of coordinates $x, y$ generates a photocurrent which is shared between the $X$ - and $Y$-electrode stripes. Reading these currents with, e.g., an op-amp transimpedance stage allows a subsequent subtraction of $x$ - and $y$-pairs of signals. For example, currents exiting the $x$-stripes $S_{x 2}$ and $S_{x 1}$ are subtracted to yield an $X$-coordinate signal $\xi=S_{x 2}-S_{x 1}$.

Due to the sharing mechanism, the obtained signal has a linear dependence on the spot coordinate. If the spot has a finite width, a distribution of coordinates will be obtained from each detected photon, and therefore, the $x$ and $y$ distribution of photons will be replicated as an output distribution of coordinates.

As for the Q-PD, let us consider the problem as monodimensional and assume that the power distribution $p(x)$ is a Gaussian of rms width (or standard deviation) $w$. Now, we can repeat the same arguments as those developed to write (4)-(7). The coordinate signal is generated as current difference; hence, it can be written as

$$
\xi=P_{0}(2 \pi)^{-1 / 2} w^{-1} 2 x_{0}
$$

whereas (5) is still valid.

About amplitude variance, the difference of currents (in $x$ or $y$ ) has a variance given by the sum of the corresponding terms, and the total is once again $2 h \nu P_{0} B$. Thus, we can conclude that (8a) and (8b) still hold for the quantum-noise limit of position uncertainty measured by the PSD.

As a first-hand estimate of the quantum limit, let us consider a $w=1$-mm spot shedding $10 \mu \mathrm{W}$ of power in the visible $\left(h \nu \approx 10^{-19} \mathrm{~J}\right)$ on a Q-PD or a PSD. Let the measurement time be $1 \mu \mathrm{s}$ (or, bandwidth be $0.5 \mathrm{MHz}$ ). Then, (8) gives the uncertainty as $\sigma_{x}=0.1 \mu \mathrm{m}$. Figures comparable to this have been actually reported as experimental result [6].

A final comment is about the possibility of improving the quantum-limited uncertainty through the use of squeezed-state 
radiation. The hint is that the squeezing factor $F$ may improve the $\mathrm{S} / \mathrm{N}$ ratio and, hence, position uncertainty. However, as noted in [7] for the Q-PD, since the mechanism involved in both the Q-PD and the PSD is current (or photon) sharing, the squeezing factor becomes spoiled by the partitioning statistics [16], and no appreciable improvement is actually obtained.

\section{B. Effect of Dark Current and Thermal Noises}

Usually, in a real experiment or measurement apparatus, the quantum limit is not easy to approach because of the many sources of nonidealities that are unintentionally superposed, often yielding noises or disturbances on the order of magnitude larger than the quantum limit.

However, when we carefully control and eliminate the extra incidental disturbances, we are left with two basic contributions additional to the quantum noise, which determines the total position variance. They are the following:

1) dark (or equivalent dark) current $I_{d}$ of the photodiode;

2) resistance (or equivalent resistance) $R$ of the preamplifier.

The first contribution summarizes in a single term $I_{d}$ all the dc currents added to the useful signal, due to the following: 1) actual dark current of the detector; 2) current due to background radiation reaching the detector; and 3) any other bias current added to detector signal. The noise contribution associated to $I_{d}$ can be described by a shot-noise [5] current variance given by

$$
\sigma_{i}^{2}=2 e I_{d} B
$$

where $B$ is the bandwidth of measurement.

The second contribution summarizes in a resistance value $R$ all the dissipative effects found in parallel to the signal line and due, e.g., to the following: 1) the input resistance of the preamplifier; 2) the differential resistance across detector terminals; and 3) the excess noise of the preamplifier. Thus, as associated with $R$, we shall consider the well-known Johnson noise [5] term. Expressed as a current-noise generator at the input node, the current variance of the noise is written as

$$
\sigma_{R}^{2}=4 k T B / R
$$

where $k$ is Boltzmann constant, and $T$ is the absolute temperature.

To include the variances of (10) and (10a) into the quantum noise result, we shall go back to the signal at the level of photo-detected currents, where they can be added as statistically independent terms. The detected current following power $P_{0}$ is $I_{0}=(\eta e / h \nu) P_{0}$, and the shot noise associated with $I_{0}$ is

$$
\sigma_{I_{o}}^{2}=2 e I_{0} B
$$

Adding the new terms (10) and (10a) yields for the total noise

$$
\sigma_{\text {tot }}^{2}=\sigma_{I_{o}}^{2}+\sigma_{i}^{2}+\sigma_{R}^{2}=2 e I_{0} B+2 e I_{d} B+4 k T B / R .
$$

Now, we can introduce an equivalent current $I_{\text {eq }}$ to summarize in a single parameter both dark current and preamplifier noise, which is defined as

$$
I_{\mathrm{eq}}=I_{d}+(2 k T / e) / R .
$$

Noting that $2 k T / e \approx 50 \mathrm{mV}$ at ambient temperature $(T=$ $300 \mathrm{~K}$ ), the second term is normally the larger (except for unusually large values of resistance). Current $I_{\mathrm{eq}}$ is also called the break-point current between quantum and thermal regimes of photo detection (see [5, Ch. 3]).

With the position of (13), the current variance is written as

$$
\sigma_{\text {tot }}^{2}=2 e I_{0} B\left(1+I_{\text {eq }} / I_{0}\right) .
$$

Now, we can go back to (5) and rewrite the uncertainty of position sorting with the photodetector as

$$
\sigma_{x}^{2}=\sigma_{\text {tot }}^{2} /\left|\partial I_{0} / \partial x\right|^{2} .
$$

Here, as $I_{0}=(\eta e / h \nu) P_{0}$ and $I(x) \approx(\eta e / h \nu) P_{0}(2 \pi)^{-1 / 2}$ $w^{-1} 2 x_{0}$, we get

$$
\begin{aligned}
\sigma_{x}^{2} & =2 e I_{0} B\left(1+I_{\mathrm{eq}} / I_{0}\right) / I_{0}^{2}(\pi / 2)^{-1} w^{-2} \\
& =w^{2}(\pi / 2) 2 e B\left(1+I_{\mathrm{eq}} / I_{0}\right) / I_{0} .
\end{aligned}
$$

Again, we note that $I_{0} / e$ is the rate at which photoelectrons are generated following photon detection. If we multiply this quantity by the observation time $T$, we get the number of electrons $N_{\mathrm{el}}=T I_{0} / e$ utilized in the measurement (or the number of actually detected photons $N_{\mathrm{ph}}$ ). Rearranging (16), we get in the general case the following equation:

$$
\sigma_{x}=(\pi / 2)^{1 / 2}\left[w / \sqrt{ } N_{\mathrm{ph}}\right] \sqrt{ }\left(1+I_{\text {eq }} / I_{0}\right) .
$$

This result tells us that dark current and amplifier noise do not change the basic dependence of uncertainty from a characteristic length and the square root of detected photons. The contributed excess noise is summarized by a multiplying factor equal to $\sqrt{ }\left(1+I_{\text {eq }} / I_{0}\right)$.

Going to specific devices, we may remark that the dark currents in the Q-PD and the PSD may be on the same order of magnitude if structure, geometry, and doping are the same. However, the PSD has an additional resistance to be accounted for: the series resistance $R_{s}$ (Fig. 2) used for current sharing between electrodes. Considering typical values of the dark current (e.g., $1 \mathrm{nA}$ ) and $R_{s}$ (e.g., $100 \mathrm{k} \Omega$ ), the latter may be the major contribution to $I_{\mathrm{eq}}$.

\section{Distance- OR DisPlacement- MEASURING INSTRUMENTS}

We can classify these instruments as follows: 1) incoherent or amplitude modulated and 2) coherent or phase measuring [4].

In the incoherent class, we find the well-known pulsed rangefinder (or laser telemeter) and the sine-wave modulated (or laser topograph) as well as the optical rule [5] and the triangulation telemeter [4]. 

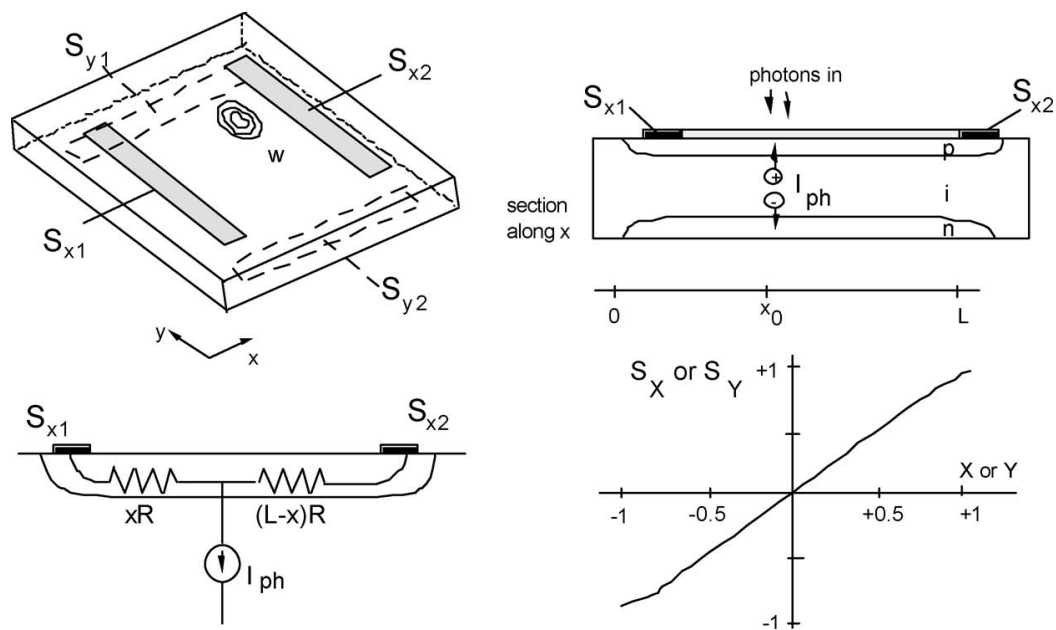

Fig. 2. Typical structure of a PSD (top left) detecting a light spot $w$. Light received at point $x, y$ generates a photocurrent $I_{\mathrm{ph}}$ that is shared between the $X$ and $Y$ electrodes (top right and bottom left). Coordinate signals are obtained as the differences between the currents exiting from the electrodes $S_{x 2}-S_{x 1}$ and $S_{y 2}-S_{y 1}$. To ensure good linearity and a negligible dependence from series resistance $R_{s}$, currents are sunk by the virtual ground of an op-amp stage.

In the first two instruments, a suitable waveform is impressed as an amplitude modulation onto the optical power carrier, for example, $s(t)$, and the measurement is performed by looking at the returning waveform $s(t-T)$, which is delayed from the propagation time $T=2 L / c$ to the remote target at the distance $L$ and back.

\section{A. Pulsed Telemeter}

Here, we summarize the derivation developed in [4]. In the pulsed telemeter, the photo-detected current is parameterized by writing it as $i=\varsigma\left(E_{r} / \tau\right) s(t / \tau)$, where $E_{r}$ is the total energy (Joule) contained in the pulse, $\varsigma$ being the spectral sensitivity of the detector (in amperes per watt), and $\tau$ (in seconds) is the time-scale factor of the waveform. We further assume that $\tau^{-1} s(t / \tau)$ is normalized to unit area, and we let $\kappa$ represent the time-bandwidth product [or, equivalently, where $\kappa / \tau$ is the 3 -dB frequency cutoff of the Fourier transform of $s(t / \tau)]$.

Then, we can write the amplitude variance associated with the shot (or quantum) noise of the detected photoelectrons as

$$
\sigma_{i}^{2}(t)=2 e \varsigma E_{r} \tau^{-1} s(t / \tau) B
$$

Now, the most obvious way to perform the timing measurement is threshold crossing (see Fig. 3) of the pulse at a suitable level $S_{0}$. With this method, the time variance $\sigma_{t}^{2}$ is readily given by (5), particularized to the case at hand, by changing $x$ and $\xi$ into $t$ and $I$, so that

$$
\sigma_{t}^{2}=\sigma_{I}^{2} /|\partial I / \partial t|^{2}
$$

By computing the slope $\partial I / \partial t$ and inserting in (19) with $B=$ $\kappa / \tau$, we readily get the following equation:

$$
\sigma_{t}^{2}=\left(\tau^{2} / E_{r}\right) 2 e \kappa S_{0} / \varsigma s^{\prime 2}
$$

where, explicitly, the time derivative $s^{\prime}=d s / d t$ is calculated at time $t_{0}$ of crossing, where $s\left(t_{0} / \tau\right)=S_{0}$. Now, we may note that the spectral sensitivity can be expressed as $\varsigma=e \eta / h \nu, \eta$ being the quantum efficiency of the detector, and that $E_{r} / h \nu$

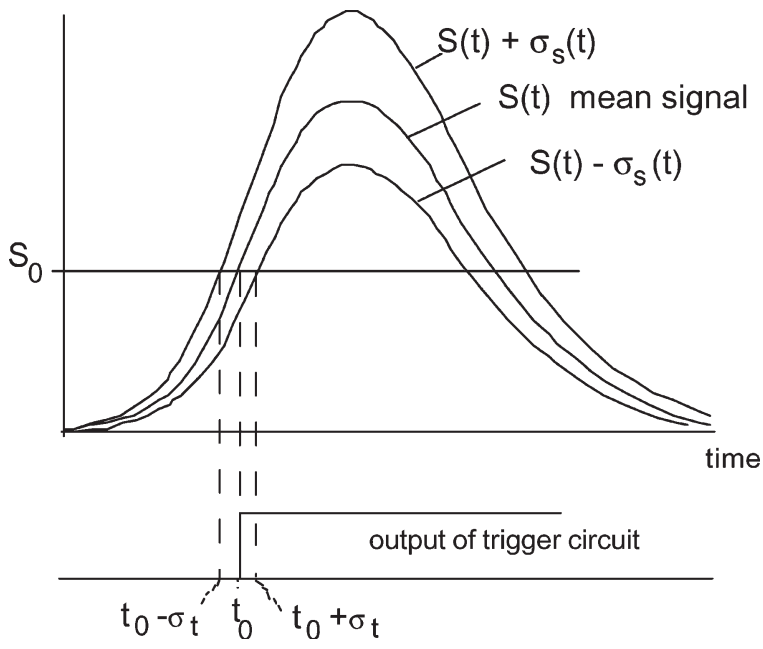

Fig. 3. Timing of the pulsed telemeter by threshold crossing with an amplitude discriminator placed at a level $S_{0}$. Time $t_{0}$ is affected by fluctuations $\pm \sigma_{t}$ due to the fluctuations in amplitude $\pm \sigma_{S}$.

is the number of photoelectrons $N_{\mathrm{ph}}$ made available in the measurement process. By using these values in (20), we easily get the following equation:

$$
\sigma_{t}=m_{f}\left(\tau / \sqrt{ } N_{\mathrm{ph}}\right)
$$

where $m_{f}=\sqrt{ }\left(2 \kappa S_{0} / s^{\prime 2}\right)$ is a number of the order of unity that summarizes the details of threshold crossing (see [4] for further details about optimum timing).

Another equivalent expression follows from (21), recalling that $\sqrt{ } N_{\mathrm{ph}}$ is also the signal-to-noise ratio of an amplitude measurement at the crossing time, i.e., $\mathrm{S} / \mathrm{N}=I_{\mathrm{ph}} / 2 e B$, where

$$
\sigma_{t}=m_{f} \tau /(\mathrm{S} / \mathrm{N}) .
$$

Finally, we can write the uncertainty $\sigma_{L}$ of distance in a timeof-flight measurement with a pulse of duration $\tau$ on a round-trip time $T=2 L / c$ as

$$
\sigma_{L}=(c / 2) \sigma_{t}=m_{f}(c / 2) \tau / \sqrt{ } N_{\mathrm{ph}}=m_{\mathrm{f} 1} L_{c} / \sqrt{ } N_{\mathrm{ph}}
$$




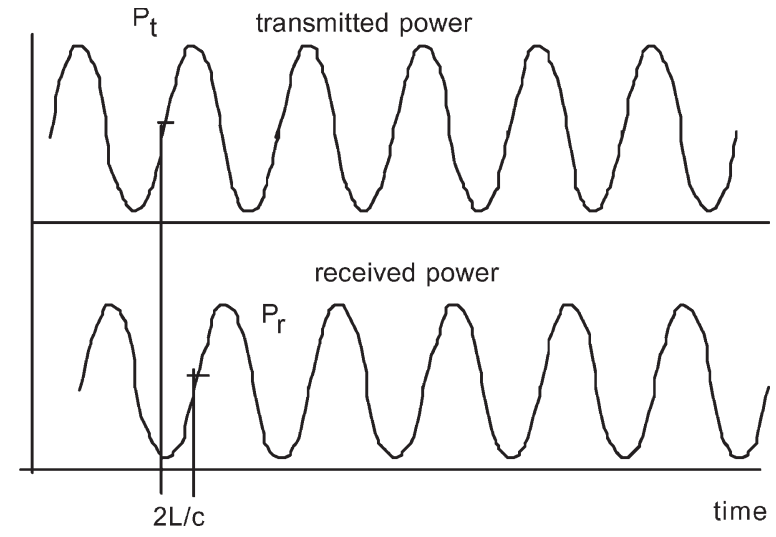

Fig. 4. Waveforms of the transmitted and received powers in a sine-wavemodulated telemeter.

where $L_{c}=c \tau / 2$ is the characteristic length describing the light pulse envelope, and the multiplicative factor is given by $m_{\mathrm{f} 1}=\left(2 \kappa S_{0}\right)^{1 / 2} / s^{\prime}$.

As an illustration, we may consider a pulsed range finder with a Gaussian pulse of the type $s(t / \tau)=$ $(2 \pi)^{-1 / 2} \tau^{-1} \exp -\left(s^{2} / 2 \tau^{2}\right)$. By looking for the minimum $\sigma_{t}^{2}$ with respect to the threshold level $S_{0}$, we can find [4] that the optimum level for placement of the threshold is $S_{0}=0.147$. Furthermore, for $\eta=1$, we obtain also (see [4] for details) $m_{\mathrm{f} 1}=0.937$.

Finally, the optimum filter handling of the Gaussian pulse is found [4] to give a substantial improvement, with $\sigma_{t}=$ $\tau / \sqrt{ } N_{\mathrm{ph}}$ as per (21), and $m_{\mathrm{f} 1}=0.506$, which is a value about half that of the fixed-threshold crossing.

\section{B. Sine-Wave-Modulated Telemeter}

In this telemeter, we transmit a signal of the form $P_{t}=$ $P_{t 0}\left(1+m \cos 2 \pi f_{m} t\right)$, where $m$ is the modulation index, and receive a delayed replica $P_{r}=P_{r 0}\left[1+m \cos 2 \pi f_{m}(t-T)\right]$ (see Fig. 4), $T=2 L / c$ being the go-and-return time of propagation to the target.

The phase shift between the two waveforms $2 \pi f_{m} T=$ $2 \pi f_{m} 2 L / c$ is the term containing the desired distance information.

Once again, by applying the above concepts and, in particular, (18) and (19), we can easily arrive at the following result describing the timing variance of the measurement process (see [4] for details):

$$
\sigma_{t}^{2}=\left(2 \pi f_{m}\right)^{-2}\left(E_{r} m / h \nu\right)^{-1}
$$

or

$$
\begin{aligned}
\sigma_{t} & =m_{f}\left(2 \pi f_{m}\right)^{-1}(\mathrm{~S} / \mathrm{N})^{-1} \\
& =m_{f}\left(2 \pi f_{m}\right)^{-1} / \sqrt{ } N_{\mathrm{ph}}
\end{aligned}
$$

where $m_{f}=m^{-1 / 2}$ is the multiplicative factor that is not much different from unity.

Equation (23) tells us that, other quantities being equal, the sine-wave and the pulsed telemeters have the same uncertainty if $\tau$ and $\left(2 \pi f_{m}\right)^{-1}$ are the same.

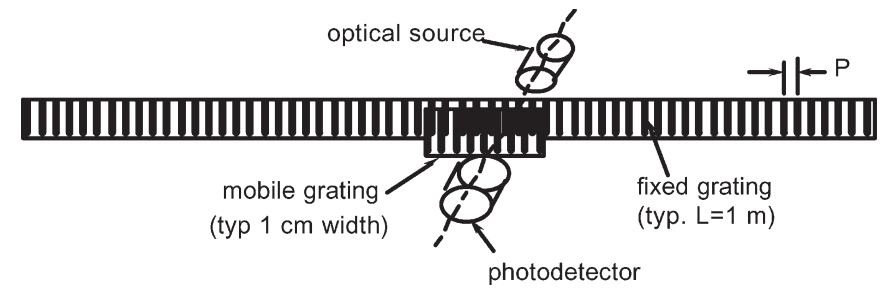

Fig. 5. Readout of position in an optical rule: The mobile grating, sliding over the fixed grating, generates a periodic variation of transmission sensed by the source and photodetector combination.

We can also put (23) in the format of (22) by letting $L_{c}=c / 2 f_{m}$ so that the characteristic length is the period of amplitude modulation of the sine wave impressed on the beam intensity, and the distance uncertainty can be written as

$$
\sigma_{L}=m_{f 2} L_{c}(\mathrm{~S} / \mathrm{N})^{-1}=m_{f 2} L_{c} / \sqrt{ } N_{\mathrm{ph}}
$$

where $m_{f 2}=\pi^{-1} m^{-1 / 2}$.

\section{Extension to Thermal Regime}

The quantum-noise limits expressed by (21)-(24) can be now extended to the case of nonnegligible dark current and termination noise, as outlined in Section II-B. In the same way, by repeating the same reasoning, we can easily see that when the thermal and dark current noises are summarized by the equivalent current $I_{\text {eq }}$ defined as in (13); we then have for the time uncertainty of the pulsed and sine-wave telemeters

$$
\begin{aligned}
\sigma_{t} & =m_{f}\left(\tau / \sqrt{ } N_{\mathrm{ph}}\right) \sqrt{ }\left(1+I_{\mathrm{eq}} / I_{0}\right) \quad(\text { pulsed }) \\
\sigma_{t} & =m_{f}\left(2 \pi f_{m} \sqrt{ } N_{\mathrm{ph}}\right)^{-1} \sqrt{ }\left(1+I_{\mathrm{eq}} / I_{0}\right) \quad \text { (sine-wave) }
\end{aligned}
$$

and similarly for the distance uncertainty given by (22) and (24).

\section{Optical Rule}

The optical rule [5] is a displacement-measuring apparatus exploiting the Moire' (also called undersampling) effect, which is generated when we look through two finely photoengraved grating with alternate transparent and opaque lines (Fig. 5). When we move, transversally, the mobile grating with respect to the fixed one, the transmitted signal obtained at the photodetector is a periodic signal of the form:

$$
I_{\mathrm{ph}}=I_{0}(1+\sin 2 \pi s / p)
$$

where $p$ is the period of the grating, and $I_{0}=\varsigma P_{0}$ is the photocurrent supplied by the LED/detector combination mounted in front of the grating for readout [5].

In (26), for simplicity, we assume the periodic dependence on displacement a sine function, but of course, this function might as well be a triangular or a distorted sinusoid, depending on the engraving profile of each line in the grating. The actual function is not critical, as we usually count the semiperiods of the signal. About the ultimate limit of uncertainty, it can be accounted for by an appropriate multiplying factor $m_{f}$ in the equations to follow. 


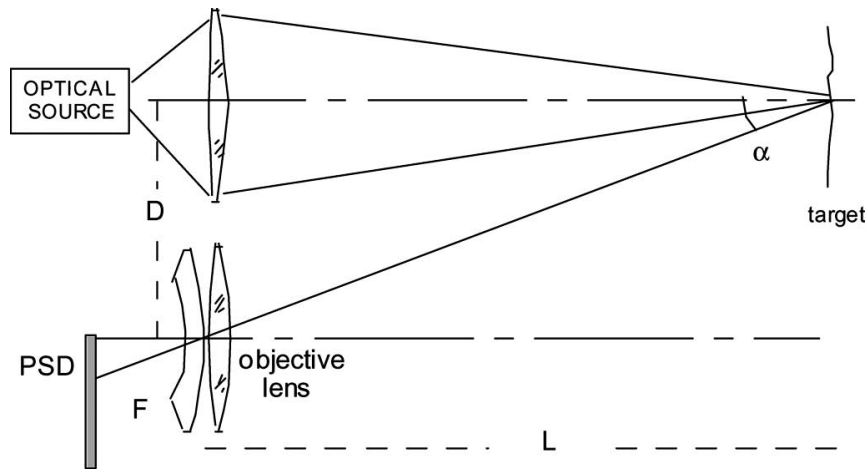

Fig. 6. In a triangulation telemeter, a one-axis PSD is used to sense the parallax angle $\alpha$ of a base $D$ on the distance under measurement $L$.

The quantum noise current associated to the detected signal is, as usual, $i_{n}^{2}=2 e I_{0} B$, whereas the minimum measurable displacement $\Delta s$ is obtained from (26) as that corresponding to a current variation $\Delta I_{\mathrm{ph}}=I_{0} 2 \pi \Delta s / p$.

By squaring $\Delta s$, averaging, and equating to $\sigma_{s}^{2}$, we obtain the positioning variance as

$$
\sigma_{s}^{2}=2 e I_{0} B\left(p / 2 \pi I_{0}\right)^{2}=(p / 2 \pi)^{2}(\mathrm{~S} / \mathrm{N})^{-2}
$$

where, the uncertainty is proportional to the period $P$ of the grating and inversely to the $\mathrm{S} / \mathrm{N}$ ratio or the square root of photoelectrons:

$$
\sigma_{s}=(p / 2 \pi)(\mathrm{S} / \mathrm{N})^{-1}=m_{f} L_{c} / \sqrt{ } N_{\mathrm{ph}}
$$

with $L_{c}=p$ and $m_{f}=1 / 2 \pi$. The extension corresponding to (25) is readily seen, also in this case, to be obtained as

$$
\begin{aligned}
\sigma_{s} & =m_{f} P(\mathrm{~S} / \mathrm{N})^{-1} \sqrt{ }\left(1+I_{\mathrm{eq}} / I_{0}\right) \\
& =m_{f}\left(L_{c} / \sqrt{ } N_{\mathrm{ph}}\right) \sqrt{ }\left(1+I_{\mathrm{eq}} / I_{0}\right) .
\end{aligned}
$$

\section{E. Triangulation Telemeter}

This simple telemeter (Fig. 6) is based on the measurement of the parallax angle that a base $D$ present at the distance to be measured. A simple analysis (see [4, Ch. 3]) shows that the uncertainty of the distance is related to the uncertainty of the angle by the relation

$$
\sigma_{L}=\left(L^{2} / D\right) \sigma_{\alpha}
$$

On its turn, the angle (and its uncertainty) is connected to the position (and its uncertainty) of the optical spot focused by the objective lens onto the PSD entrance surface as

$$
\sigma_{x}=\sigma_{\alpha} F
$$

under the tacit assumption of small parallax $(\tan \alpha \approx \alpha)$. Using (8) or (8b) for the uncertainty of the coordinate measured by the PSD, and (30), readily yields the distance uncertainty as

$$
\begin{aligned}
\sigma_{L} & =\left(L^{2} / D F\right) \sigma_{x} \\
& =(\pi / 2)^{1 / 2}\left(w L^{2} / D F\right) \sqrt{ } N_{\mathrm{ph}} \\
& =m_{f}\left(L_{c} / \sqrt{ } N_{\mathrm{ph}}\right)
\end{aligned}
$$

where the last expression of the line tells us that, once again, the dependence is from length divided by square root of photons and that, in this case, $L_{c}=w L^{2} / D F$, and $m_{f}=(\pi / 2)^{1 / 2}$.

\section{F. Coherent Measurements}

Coherent displacement measurements are exemplified by the well-known laser interferometers [4]. In these instruments, irrespective of the optical configuration used for defining the optical arms and the splitting and recombining arrangement, the interference signal generated at the photodetector can be written in the form

$$
I_{\mathrm{ph}}=I_{0}[1+V \cos (R k s+\Phi)] .
$$

Here, $k=2 \pi / \lambda$ is the wavenumber, $s$ is the imbalance of interferometer arms or the displacement to be measured, $I_{0}$ is the average current at the photodetector, $V$ is fringe visibility (or coherence factor), and $R$ is the responsivity of the optical interferometer ( $=2$ for a Fabry-Pérot and $=1$ for the Mach-Zehnder, etc.; see [4]).

Current $I_{\mathrm{ph}}$ is affected by the shot noise with a quadratic mean value $i_{n}^{2}=2 e I_{0} B$, and the distance or displacement $s$ is best measured when the interferometer signal is biased at half-fringe or for $\phi=\pi / 2$. By substituting in (30) and with the approximation $\sin \Psi \approx \Psi$, we readily get for the variance $\sigma_{s}^{2}$ of the displacement the following equation:

$$
\sigma_{s}^{2}=\left(2 e B / I_{0}\right)(V R k)^{-2}=(\lambda / 2 \pi V R)^{2}(\mathrm{~S} / \mathrm{N})^{-2}
$$

Or

$$
\sigma_{s}=m_{f}\left(L_{c} / \sqrt{ } N_{\mathrm{ph}}\right)
$$

where $L_{c}=\lambda$, and $m_{f}=(2 \pi V R)^{-1}$. Once again, we find the dependence of uncertainty as a length divided by the number of photons used in the measurement. It is worth noting that this time for $L_{c}$, we get $\lambda$, that is, the smallest length that is physically allowed by the experiment and a quantity that is by far much smaller than in all other cases considered above. Yet, the consequent much increased sensitivity is paid by the feature of interferometric measurement being incremental-different from a laser telemeter measurement, which is absolute. As it is well known, this is why we call the measurements performed with the laser interferometer a displacement measurement opposed to the distance measurement of a telemeter.

Recently [14], [15], it has been reported that an interferometric scheme can also be employed in an absolute distance measurement, taking advantage of the synthetic wavelength $\lambda_{\text {syn }}$, which comes about when the optical wavelength is swept from an initial value $\lambda_{0}$ to a final value $\lambda_{0}+\Delta \lambda$. An analysis of the problem [4] reveals that (34) still holds in this case, provided that we use in it the synthetic wavelength $\lambda_{\text {syn }}=\lambda_{0}^{2} / \Delta \lambda$ in place of $\lambda$.

The coherent measurement has the special interesting feature of being practically unaffected by thermal and dark current noises. Indeed, as the coherent superposition of the reference and measurement field at the photodetector is just the process of coherent detection [4], [5], as opposed to direct detection of 
TABLE I

Characteristic Length of Position And Displacement-or DisTANCE-MEASURING DeVICES

\begin{tabular}{lcc}
\hline Device/Instrument & $\mathrm{L}_{\mathrm{c}}$ - main dependence & $\mathrm{m}_{\mathrm{f}}$ - multiplying factor \\
Quadrant Photodiode & $\mathrm{w}$ & $(\pi / 2)^{1 / 2}$ \\
PSD (position sensing PD) & $\mathrm{w}$ & $(\pi / 2)^{1 / 2}$ \\
Optical rule & $\mathrm{p}$ & $(2 \pi)^{-1}$ \\
Pulsed Telemeter & $\mathrm{c} \tau / 2$ & $\left(2 \mathrm{KS}_{0}\right)^{1 / 2} / \mathrm{s}$ \\
“ with Gaussian pulse & $\mathrm{c} \tau / 2$ & 0.937 \\
“ " and optimum filter & $\mathrm{c} \tau / 2$ & 0.506 \\
Sine-Wave Telemeter & $\mathrm{c} / 2 \mathrm{f}_{\mathrm{m}}$ & $\pi^{-1} \mathrm{~m}^{-1 / 2}$ \\
Triangulation telemeter & $\mathrm{wL}^{2} / \mathrm{DF}$ & $(\pi / 2)^{1 / 2}$ \\
Laser Interferometer & $\lambda$ & $(2 \pi \mathrm{VR})^{1}$ \\
$\lambda$-swept Distance Meter & $\lambda_{0}{ }^{2} / \Delta \lambda$ & $(2 \pi \mathrm{V})^{1}$ \\
\hline
\end{tabular}

Caption: $w=$ spot size of a Gaussian distribution; $p=$ period of optical rule; $\tau=$ duration of the pulse and $\kappa$ its time-bandwidth product; $S_{0}=$ threshold of timing (Fig.3); $s^{\prime}=$ normalized signal slope at threshold; $f_{m}=$ modulation frequency of sinewave; $m=$ modulation index; L,D,F distance, base, focal length of triangulation (Fig.6); V=fringe visibility, $\mathrm{R}=$ interferometer responsivity (=2 for a Fabry-Pérot)

other schemes, we should theoretically multiply again the displacement uncertainty by a factor of the form $\sqrt{ }\left(1+I_{\text {eq }} / I_{0}\right)$, but now, $I_{0}$ is no more the average signal; rather, it is the local oscillator average current [4], [5]. By working at a sufficiently high level, or $I_{0} \gg I_{\text {eq }}$, we can make the thermal and dark current noise contributions negligible at all times.

\section{CONCLUSION}

We have derived the uncertainty of positioning devices at the quantum noise limit and found that the main dependence is from the inverse square root of the number $\sqrt{ } N_{\text {ph }}$ of photoelectrons detected in the measurement time interval or from the signal-to-noise ratio $\mathrm{S} / \mathrm{N}=\left[I_{0} / 2 e B\right]^{1 / 2}$ of the mean photocurrent by which the measurement is performed. In addition, the multiplication factor $m_{f}$ to the main dependence $L_{c} / \sqrt{ } N_{\mathrm{ph}}$ is on the order of unity, and it has been evaluated for a number of position-sense and displacement- or distancemeasuring arrangements.

In Table I, we summarize the results of our analysis about characteristic length $L_{c}$ and multiplication factor $m_{f}$ at the quantum limit.

Last, we have found that when the device deviates from the quantum limit, the worsening of uncertainty is easily described by the excess factor $\sqrt{ }\left(1+I_{\mathrm{eq}} / I_{0}\right)$, in which $I_{\mathrm{eq}}$ is the equivalent current, describing thermal noise of the photodetector load resistance and the dark current noise.

\section{REFERENCES}

[1] "Special issue on 'Optical distance measurements and applications'," J. Opt. A, vol. 4, no. 6, pp. S281-S420, Nov. 2002. T. Bosch, and S. Donati, Eds.

[2] H. Lamela, Ed., Proc. ODIMAP V, Madrid, Spain: LEOS Spanish Chapter, Oct. 2-6, 2006.

[3] R. D. Hudson, Jr., Infrared System Engineering. New York: WileyInterscience, 1969, ch. 3.

[4] S. Donati, Electrooptical Instrumentation. Upper Saddle River, NJ: Prentice-Hall, 2004, ch. 2.2

[5] S. Donati, Photodetectors. Upper Saddle River: Prentice-Hall, 2000, ch. 9.3.4. Section 8.6.

[6] C. A. J. Putman, B. G. De Grooth, N. F. Van Hulst, and J. Greve, "A detailed analysis of the optical beam deflection technique for use in atomic force microscopy," J. Appl. Phys., vol. 72, no. 1, pp. 6-12, Jul. 1992.
[7] C. Fabre, J. B. Fouet, and A. Mitre, "Quantum limits in the measurement of very small displacements in optical images," Opt. Lett., vol. 25, no. 1, pp. 76-79, Jan. 2000.

[8] A. Makynen, J. T. Kostamovaara, and R. A. Myllyla, "Displacement sensing resolution of position-sensitive detectors in atmospheric turbulence using retroreflected beam," IEEE Trans. Instrum. Meas., vol. 46, no. 5, pp. 1133-1136, Oct. 1997.

[9] A. Makynen, T. Kostamovaara, and R. A. Myllyla, "A high-resolution lateral displacement sensing method using active illumination of a cooperative target and a focused four-quadrant position-sensitive detector," IEEE Trans Instrum. Meas., vol. 44, no. 1, pp. 46-52, Feb. 1995.

[10] A. Makynen, J. Kostamovaara, and R. Myllyla, "Positioning resolution of the position-sensitive detectors in high background illumination," IEEE Trans. Instrum. Meas., vol. 45, no. 1, pp. 324-326, Feb. 1996.

[11] A. Rohrbach, H. Kress, and E. H. K. Stelzer, "Three-dimensional tracking of small spheres in focused laser beams: Influence of the detection angular aperture," Opt. Lett., vol. 28, no. 6, pp. 411-413, Mar. 2003.

[12] M. Toyoda, K. Araki, and Y. Suzuki, "Wave-front tilt sensor with two quadrant detectors and its application to a laser beam pointing system," Appl. Opt., vol. 41, no. 12, pp. 2219-2223, Apr. 2002.

[13] A. Papoulis, Probability, Random Variables and Stochastic Processes. New York: McGraw-Hill, 1965.

[14] T. Bosch, N. Servagent, and S. Donati, "Optical feedback interferometry for sensing applications," Opt. Eng., vol. 40, no. 1, pp. 20-27, 2001.

[15] M. Norgia, G. Giuliani, and S. Donati, "New absolute distance interferometric technique," in Proc. SPIE Conf. Opt. Metrology Production Eng., W. Osten and M. Takeda, Eds., Strasbourg, France, Apr. 27-30, 2004, vol. 5457, pp. 423-431.

[16] V. Annovazzi Lodi, S. Donati, and S. Merlo, "Squeezed states in direct and coherent detection," Opt. Quantum Electron., vol. 24, no. 3, pp. 285-301, 1992.

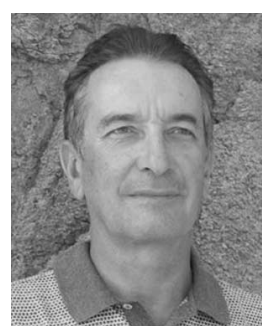

Silvano Donati (F'03) graduated in physics cum laude from the University of Milano, Milan, Italy, in 1966.

He has been at the University of Pavia, Pavia, Italy, since 1980, where he is currently a Full Professor of photodetectors and electrooptical instrumentation, which are topics that have been covered by his homonymous books published by Prentice-Hall in 2000 and 2004. His main research has been on photodetectors, electrooptical instrumentation (particularly selfmix interferometry), and nonlinear dynamic effects in semiconductor lasers (in particular, chaos cryptography). His seminal papers have gotten more than 500 citations. He has authored or coauthored over 250 papers in international journals, and he is the holder of 12 patents.

Prof. Donati is a Fellow of OSA and a meritorious member of AEI. In 1997, he founded the IEEE-LEOS Italian Chapter and chaired it until 2002. He served LEOS as Vice President of Membership Region 8 from 2002 to 2004, and currently, he is a member of the LEOS Board of Governors. He has chaired national and international meetings like Elettroottica (Pavia, Italy, 1994 and 2004), Fotonica (Roma, Italy, 1997) of the National Association AEI, the WFOPC International Conference in 1998 and 2000, and ODIMAP in 1999 and 2001, which was organized by the IEEE-LEOS

Cheng-Yen Chen, photograph and biography not available at the time of publication.

Chih-Chung Yang (S'81-M'83-SM'94), photograph and biography not available at the time of publication. 